\title{
Characterization of female intimate hygiene practices and vulvar health: A randomized double-blind controlled trial
}

Filippo Murina MD, Camilla Caimi MD, Raffaele Felice MD, Stefania Di Francesco MD, Irene Cetin MD

Lower Genital Tract Disease Unit, V. Buzzi Hospital-University of Milan, Milan, Italy.

\section{Funding information:}

This study was supported by the nonprofit Italian association Fondazione Ricerca Donna e Feto.

\begin{abstract}
Background

Inappropriate feminine hygiene practices are related to vulvar unpleasant symptoms (such as skin changes, lesions, burning, pruritus, fissures, and dyspareunia).
\end{abstract}

Aims

We assessed the daily use effects of intimate cleansers on vulvar skin by comparing two specific products for intimate care: Saugella Hydraserum (SIS), based on natural extracts, and a standard product based on lactic acid, such as Lactacyd Feminine Hygiene (LTC). Forty healthy women were enrolled in this double-blind controlled study.

Methods

After randomization, the cleansers were used twice daily for 30 days. The hydration level was determined using the Corneometer ${ }^{\circledR}$ CM 825, the pH using the Skin-pH-Meter PH $905^{\circledR}$ and the sebum level using the Sebumeter SM815 ${ }^{\circledR}$. Measurements were performed at baseline and on day 30 on the labia majora and labia minora.

Results

Both cleansers showed a reduction in the hydration level, but this was much less evident in the SIS group ( $-6.3 \%$ SIS vs $-23,7 \%$ LTC). The $\mathrm{pH}$ values of the SIS group were lower than those of the LTC group, especially on the labia minora (5.27 \pm 0.08 and $5.6 \pm 0.1$, respectively, $P=.025)$. The sebum increased in both groups, but in the LTC group, it was higher on the labia majora $(+96.2 \% \mathrm{vs}+46.8 \%$, respectively, $P=.003)$, while on the labia minora, it was higher in the SIS group $(+24.7 \% \mathrm{vs}+17.1 \%$, respectively $P=\mathrm{NS}$ ).

Conclusions 
Both cleansers tested showed high performance for safety and tolerability on vulvar skin, but SIS showed better efficacy than LTC on some parameters.

\section{INTRODUCTION}

Intimate hygiene habits may vary considerably based on several aspects, such as personal preferences, cultural norms and religious practices, and indications from healthcare professionals. However, the adoption of correct habits is crucial in preserving the entire vulvo-vaginal area health and preventing or reducing a variety of unpleasant symptoms (such as skin changes, lesions, burning, pruritus, fissures, and dyspareunia). Currently, there are many different feminine intimate hygiene products, but some may be too aggressive. For this reason, attention should be paid to choosing an intimate cleansing product according to product characteristics, its impact on $\mathrm{pH}$ or on the composition of the normal vulvo-vaginal microbiota, which are essential for protection against infection. The barrier function of vulvar skin is weaker than that at other anatomical sites, and vulvar skin has been shown to react more intensely than other skin areas to irritants.1 The hydrolipidic layer or film, which acts as a protectant, must be maintained correctly by choosing intimate care products. Moreover, compared with other areas, vulvar skin is exposed to many irritants, including occlusion, friction, sweating, vaginal secretions, and urine, which increase the susceptibility of the skin to irritant and allergic contact dermatitis. $\underline{\mathbf{2}}, \underline{\mathbf{3}}$ Intimate hygiene is a routine activity, but the differences related to the age and physiopathological condition of the woman, which influence the balance of the vaginal ecosystem, require the selection of the appropriate formulation for each individual.

Over $60 \%$ of 530 women attending a specialty clinic for vulvar diseases reported adverse intimate hygiene practices. $\underline{4}$ Irritating chemicals contained within detergents, fragrances, and lubricants can be due to irritants and allergic vulvar contact dermatitis. $\underline{1}$

Marren et al studied the correlation between patch test results of 135 patients presenting with vulvar symptoms. $\underline{5}$ Of them, 63 patients (47\%) had at least one positive reaction, with a relevant hypersensitivity related to some components related to detergents such as surfactants, preservatives, or perfumes. Most women with a vulvar disorder (eg, vulvar dermatosis, vulvovaginitis) need advice about vulvar skin care and how to avoid a worsening of symptoms and, in some circumstances, the development of disease. It was demonstrated that a specific intimate cleanser was effective in reducing burning and the concomitant emollient treatment in vulvar dermatoses. 6 Under these circumstances, the use of a nonirritant cleanser based on soft natural surfactants with hydrating and emollient properties can be a therapeutic tool. It was also shown that the occurrence of bacterial vaginosis is strongly associated with the use of commercial intimate cleansers containing antiseptic products. $\underline{7}$

It is therefore essential that healthcare professionals begin to understand the importance of vulvar skin health and how the hygiene products that they recommend impact women's bodies and genitals. 
Nevertheless, there is little published in the medical literature about intimate feminine hygiene. To close this gap, we compared the vulvar skin effects and tolerability of two intimate cleansers: Saugella Hydraserum (SIS), based on natural extracts and lactic acid, and a standard product, such as Lactacyd Feminine Hygiene (LTC).

\section{METHODS}

The study was designed as an interventional, double-blind controlled prospective trial on one cohort of healthy women. Institutional review board approval for the study was obtained.

All asymptomatic healthy women at least 18 years of age and before menopause (the absence of menstruation for 12 months) who attend our hospital for their routine gynecologic examination were invited to participate. Patients willing to participate were given informed consent documents and agreed to sign and follow the protocol. Exclusion criteria included current pregnancy or breastfeeding status, an active vulvo-vaginal infection at the time of their gynecological examination, a vulvar dermatosis or other vulvar disease, lesions in the vulva that have not been evaluated and unwillingness to wash out one's usual intimate cleanser.

Following consent to participate, all subjects underwent an evaluation of vulvar skin parameters regarding the hydration level, $\mathrm{pH}$, and sebum level of the skin surface.

The measurements were performed using the Corneometer ${ }^{\circledR}$ CM 825, Skin-pH-Meter PH $905^{\circledR}$ and Sebumeter SM815 ${ }^{\circledR}$ devices, each with a probe connected to an MDD4 base unit (Courage-Khazaka). The Corneometer ${ }^{\circledR} \mathrm{CM} 825$ measuring principle is based on a validated capacitance method. $\underline{\mathbf{8}}$ The probe (surface area of $0.95 \mathrm{~cm}^{2}$ ) contains an interdigital grid of gold electrodes covered by a low dielectric vitrified material of $20-\mu \mathrm{m}$ thickness.

A resonating system in the instrument detects the frequency shift of the oscillating system related to the capacitance (and hence hydration) of the tissue in contact with the probe.

The variable total capacitance of the skin is converted to arbitrary units (AU) of skin surface hydration, and the range of the device is 1 to $120 \mathrm{AU} . \underline{\mathbf{8}}$

The measurement of pH through the Skin-pH-Meter PH $905^{\circledR}$ device is based on a high-quality combined electrode, in which a glass electrode and an additional reference electrode are placed in one housing that is connected to a handheld probe.9 When placed on the skin, the glass electrode measures the electric potential on the surface by sensing the hydrogen ion concentration and compares it with the voltage of a known solution in the reference electrode.

The potential difference between the two electrodes is deduced as the $\mathrm{pH}$ of the skin and is calibrated on a digital screen. $\underline{\mathbf{8}}$ The sebum level of the skin surface was determined using Sebumeter ${ }^{\circledR}$, which is based on grease spot photometry. The device contains a matt tape that becomes transparent after contact with the sebum with a measuring area of $64 \mathrm{~mm}^{2}$. After 30 seconds of skin contact with slight pressure, the system 
determines the amount of reflected light getting through the transparent area depending on the skin sebum content.

The result is expressed in $\mu \mathrm{g}$ sebum $/ \mathrm{cm}^{2}$ of skin, with a range from 0 to $350 . \underline{\mathbf{1 0}}$

We assessed sequential measurements on the middle third of the left and right sides of the labia majora and labia minora. Three repeated measures were taken at each vulvar skin site, and an average value was calculated.

All procedures were performed by the same investigator in a room under controlled temperature and humidity conditions ( $22 \pm 2^{\circ} \mathrm{C}$ and $45 \pm 5 \%$ relative humidity), monitored and recorded during the test procedures via sensors connected to the MDD4 base unit and according to the guidelines for standardized measurements. 11

Subjects meeting inclusion criteria were randomly and blindly assigned to receive Saugella HydraSerum-Mylan pharma (SIS) or Lactacyd Feminine Hygiene (LTC) through a randomization list generated by the pharmacy. Identical dispensers containing $200 \mathrm{~mL}$ of product were received from the manufacturer in separate boxes with the same color, and they were numbered randomly by staff not participating in the study in a pharmacy outside the hospital.

Investigators were blinded to the randomization code until all data were analyzed.

The composition of each test product is described in Table $\underline{\mathbf{1}}$.

Table 1. Composition of each intimate cleanser tested

\section{Product Composition}

Saugella Aqua, diglycerin, sodium cocoyl wheat amino acids, disodium laureth sulfosuccinate, Idraserum sodium lauryl sulfoacetate, xanthan gum, maltodextrin, Calendula officinalis flower extract, salvia officinalis extract, decyl glucoside, Coco-glucoside, glyceryl oleate, propylene glycol, Avena sativa extract, hydrolyzed oat protein, potassium palmitoyl hydrolyzed oat protein, lac, ketoglutaric acid, latic acid, glyceryl isostearate, glycol distearate, potassium palmitoyl hydrolyzed wheat protein, caprylyl glycol, glycerin, sodium levulinate, sodium anisate.

Lactacyd Lactoserum, lactic acid, magnesium laureth sulfate, disodium laureth sulfosuccinate, Feminine cocoamidopropyl betaine, glyceryl laurate, glycol distearate, sodium laureth sulfate, Hygiene cocamide MEA, laureth - 10, PEG-7-glyceryl cocoate, propylene glycol, 5-bromo-5nitro-1, 3-dioxane, propylene glycol, PEG-55 propylene glycol oleate, fragrance, phenoxyethyl alcohol, aqua. 
After the medical history and gynecological examination were conducted according to the randomization protocol, the women were asked to use the products as a normal detergent for feminine hygiene twice daily (morning and evening) for 30 days.

The primary endpoint for this study included the mean change from baseline to 30 days of vulvar skin parameters. Subjective evaluations of burning, rubbing, applicability, and pleasantness were also collected after 30 days, using a 4-point intensity scale $(0=$ absent, $1=$ mild, $2=$ high, $3=$ strong). The sample size of 20 subjects per group was chosen for this study based on this being a pilot study, and this sample size will be enough to prospectively document visible changes in vulvar skin parameters in this cohort of women. Quantitative variables (ie, demographic), if normally distributed, were described as the mean \pm standard deviation (SD); otherwise, the median, minimum, maximum, and interquartile range were shown.

To evaluate changes over time before and after the treatment, paired $t$ tests for dependent variables and one-way analysis of variance (ANOVA) were performed. A level of $P<.05$ was considered statistically significant. Statistical analyses were performed using SPSS (IBM SPSS statistics 21; IBM).

\section{RESULTS}

Of 45 women meeting the inclusion criteria of the study, 40 agreed to participate in the study, and 20 were blinded and randomized to receive SIS and 20 LTC. No women were lost to follow-up; thus, all 40 subjects completed the study. Participants ranged in age from 22 to 50 years, and no difference in age was noted between the SIS group (mean, $33.1 \pm 2.0$ years) and the LTC group (mean,

$32.4 \pm 2.2$ years; $P=\mathrm{NS}$ ). All women were of Caucasian origin.

After 30 days of application, both cleansers showed a reduction in the hydration level compared with the initial value, even if it was not possible to show statistically significant differences regarding specific sites of parameter collection (Figure $\underline{\mathbf{1}}$ ). However, the hydration reduction was much less evident in the SIS group, especially in the labia majora, in which the value was $-0.63 \%$ in the SIS group and $-23.7 \%$ in the LTC group, and the same tendency was shown for the labia minora (Table $\underline{\mathbf{2}}$ ). It is noteworthy to highlight that the abovementioned result represents a highly meaningful advantage from the standpoint of women's well-being, as clarified in detail in the discussion.

Table 2. Regional differences of vulvar skin parameters after treatment with SIS and LCT intimate cleanser

Group SIS Tday30-Tday0 (\%)
Group LTC

Tday30-Tday0 (\%)
$P$-value

Labia majora 


\section{Group SIS}

Hydration level

$-3.4 \pm 6.9(-6.3 \%)$

$-13.0 \pm 3.6(-23.7 \%)$

NS

$\mathrm{Ph}$

$0.3 \pm 0.1$

$0.5 \pm 0.1$

NS

Sebum level

$17.5 \pm 7.8(46.8 \%)$

$22.9 \pm 7.1(96.2 \%)$

.003

Labia minora

Hydration level

$-1.0 \pm 3.8(-0.97 \%)$

$-7.3 \pm 2.2(-6.62 \%)$

NS

$\mathrm{Ph}$

$-0.1 \pm 0.1$

$0.2 \pm 0.1$

.025

Sebum level

$12.0 \pm 7.0(24.7 \%)$

$9.3 \pm 7.7(17.1 \%)$

NS

- Mean \pm standard deviation.

- Abbreviations: LTC, Lactacyd Feminine Hygiene; NS, not significant; SIS, Saugella Hydraserum.

The mean $\mathrm{pH}$ values at the vulvar sites examined were $5.60 \sim 5.20$ for both study groups before the use of the cleansers. In the SIS group, the mean $\mathrm{pH}$ values on the labia majora and minora were significantly lower than those in the LTC group after 30 days of application, especially at the level of the labia minora (5.27 \pm 0.08 and $5.6 \pm 0.1$, respectively; $P=.025$; Figure $\underline{\mathbf{2}}$, Table $\underline{\mathbf{2}})$.

The mean amounts of regional sebum increased in both groups after 30 days of application (Figure $\underline{\mathbf{3}}$ ).

However, a strange mismatch frequency between vulvar skin types was noted in the two study groups. At 30 days, the LTC-treated group showed a statistically significant increase in the mean sebum level on the labia majora compared with the SIS group for the same area $(+96.2 \% \mathrm{vs}+46.8 \%$, respectively, $P=.003)$ (Table 2). On the labia minora instead, the SIS-treated area showed the largest percentage increase in the mean sebum level compared with the LTC group for the same area $(+24.7 \% \mathrm{vs}+17.1 \%$, respectively), but this difference was not statistically significant (Table $\underline{\mathbf{2}}$ ). All women reported that both intimate cleansers were gentle on the vulvar skin with very good tolerability. In fact, the week 4 mean burning sensation was irrelevant, even if the rubbing was significantly lower in the SIS group (1.45 \pm 0.43 vs $1.90 \pm 0.23$, respectively, $P=.003$ ) (Table $\underline{\mathbf{3}}$ ).

Table 3. Vulva skin tolerance and sensation of the intimate cleansers 


\begin{tabular}{|c|c|c|c|}
\hline Variable & SIS group $(n=20)$ & LCT group $(n=20)$ & $\boldsymbol{P}$ \\
\hline Burning & $0.95 \pm 0.47$ & $0.05 \pm 0.04$ & NS \\
\hline Rubbing & $1.45 \pm 0.43$ & $1.90 \pm 0.23$ & $P=.003$ \\
\hline Applicability & $7.90 \pm 0.59$ & $9.26 \pm 0.20$ & $P=.04$ \\
\hline Pleasantness & $7.2 \pm 0.62$ & $8.11 \pm 0.35$ & NS \\
\hline
\end{tabular}

- Mean \pm standard deviation. Questionnaires were completed after 30 days of application of the products.

- Abbreviations: LTC, Lactacyd Feminine Hygiene; NS, not significant; SIS, Saugella Hydraserum.

\section{DISCUSSION}

The vulvar skin is protected by an acidic hydrolipidic film, which possesses the properties required to maintain the skin's barrier. The skin's coating is a watery substance that is combined with oils derived by the sebaceous glands.12 These glands secrete sebum, which functions to lubricate, waterproof, and protect both the skin and hair. An ideal intimate hygiene product should first contain a mild surfactant, thus allowing for a delicate balance between skin hygiene and stratum corneum barrier damage.

Surfactant barrier damage increases transepidermal water loss and facilitates the removal of the natural moisturizing factor.

This is the mechanism by which surfactants induce the rough, scaly appearance characteristic of vulvar dermatoses.1 One important finding of our study is the minimal hydration reduction in the SIS group, especially on the labia majora, for which the value was $-6.3 \%$ compared with $-23.7 \%$ in the LTC group. This finding could be explained by the fact that SIS contains substances with relevant hydrating and emollient activity (oat milk, maltodextrins, diglycerol, and caprylyl glycol), which contribute to restoring and/or maintaining the right water content and therefore physiological skin elasticity in the external genitalia.13 Furthermore, the potential damage to the vulvar skin has been minimized using mild surfactants contained in SIS, such as derivatives of coconut and amino acids of wheat. 13 Moreover, the higher hydration reduction in the LTC group is probably due to the sodium lauryl sulfate contained in the product. Anionic surfactants, such as sodium lauryl sulfate and sodium laurate, are more irritating than other surfactants causing alterations in stratum corneum function, making it more vulnerable to injury or trauma. $\underline{14}$

Barrier damage is also influenced by intimate cleanser $\mathrm{pH}$. The important role of $\mathrm{pH}$ in maintaining skin structure and function was demonstrated by Fluhr and colleagues, $\underline{\mathbf{1 5}}$ who highlighted that small and sustained $\mathrm{pH}$ increases, such as those caused by neutral or slightly acidic intimate cleansers, adversely influence the barrier repair mechanism. 
Furthermore, an acidic $\mathrm{pH}$ in the vulvo-vestibular area is pivotal to maintaining a normal vulvo-vaginal microbiota needed for protection against infection.

In our study, we demonstrated that vulvar $\mathrm{pH}$ was stable after the use of both products tested, an essential element for an ideal intimate cleanser. The presence of lactic acid and microbiologically pure milk serum helps to maintain the correct physiological $\mathrm{pH}$, thereby fighting the development of pathogenic microorganisms. A previous study indicated that using a lactic acid plus lactoserum intimate cleanser ( pH 4-5) may help prevent the recurrence of bacterial vaginosis after oral metronidazole.16 In addition, washing the genital area more than twice a day or using different commercial products may increase the risk of infection by disturbing the genital flora and is therefore not suggested by the guidance on vulvar skin care of the Royal College of Obstetricians and Gynaecologists.17 Furthermore, using only water can cause dry skin, making the vulvar surface more vulnerable to the occurrence of itching and burning. $\underline{\mathbf{1 7}}$

It has been shown that washing vulvar skin with a harsh liquid cleanser containing an anionic amphoteric surfactant mix without any moisturizing ingredients can reduce the levels of fatty acids and cholesterol in skin even in the short term, with the impairment of healthy vulvar skin. $\underline{\mathbf{1 8}}$

The quantification of vulvar skin sebum levels was another strength of our study.

We demonstrated that the vulvar skin sebum content increases considerably after using both cleansers, which most likely resulted from the coco-glucoside and glyceryl oleate present in the products. The disparity of the sebum level between the vulvar skin types reported in the two groups is difficult to explain. In fact, the SIS-treated group showed an increase in the mean sebum level on the labia minora compared with the LTC, while on the labia majora, the measurement was the opposite. We can postulate that due to its physical properties and hydrophilic colloid nature, the xanthan gum contained in SIS ensures that the sebum-regulating and wetting agents remain in situ for a longer period, including during and after cleansing.13 This activity can be clearer on the labia minora that exhibit less keratinized epithelia than the labia majora, which is likely to be more permeable than keratinized skin. On the other hand, a greater level of sebum on the labia majora for LTC may be attributed to its features or to some bias related to sample selection.

Finally, the absence of discomfort after the use of both cleansers is evidence of the need for feminine wash products to be formulated and tested specifically for the vulvar area to ensure that they do not cause skin irritation or sensitization. Potential weaknesses of our study include the limited sample size and the opportunity to compare more products.

Nonetheless, it is worthwhile to specify that to our knowledge, this is the first clinical trial carried out with a randomized double-blind controlled design regarding the measurement of vulvar skin parameters after the use of products for intimate hygiene. 


\section{CONCLUSION}

Our study suggests that it is mandatory to choose intimate feminine hygiene products that have been clinically assessed in terms of tolerability, are pH-balanced, are hypoallergenic, and are specifically designed to help maintain natural vulvar skin homeostasis. Future research using prospective or casecontrol designs can build on our preliminary correlational findings to assess causal links between vulvar health and hygiene behaviors.

\section{CONFLICT OF INTEREST}

The authors declare no conflicts of interests. 


\section{FIGURES:}

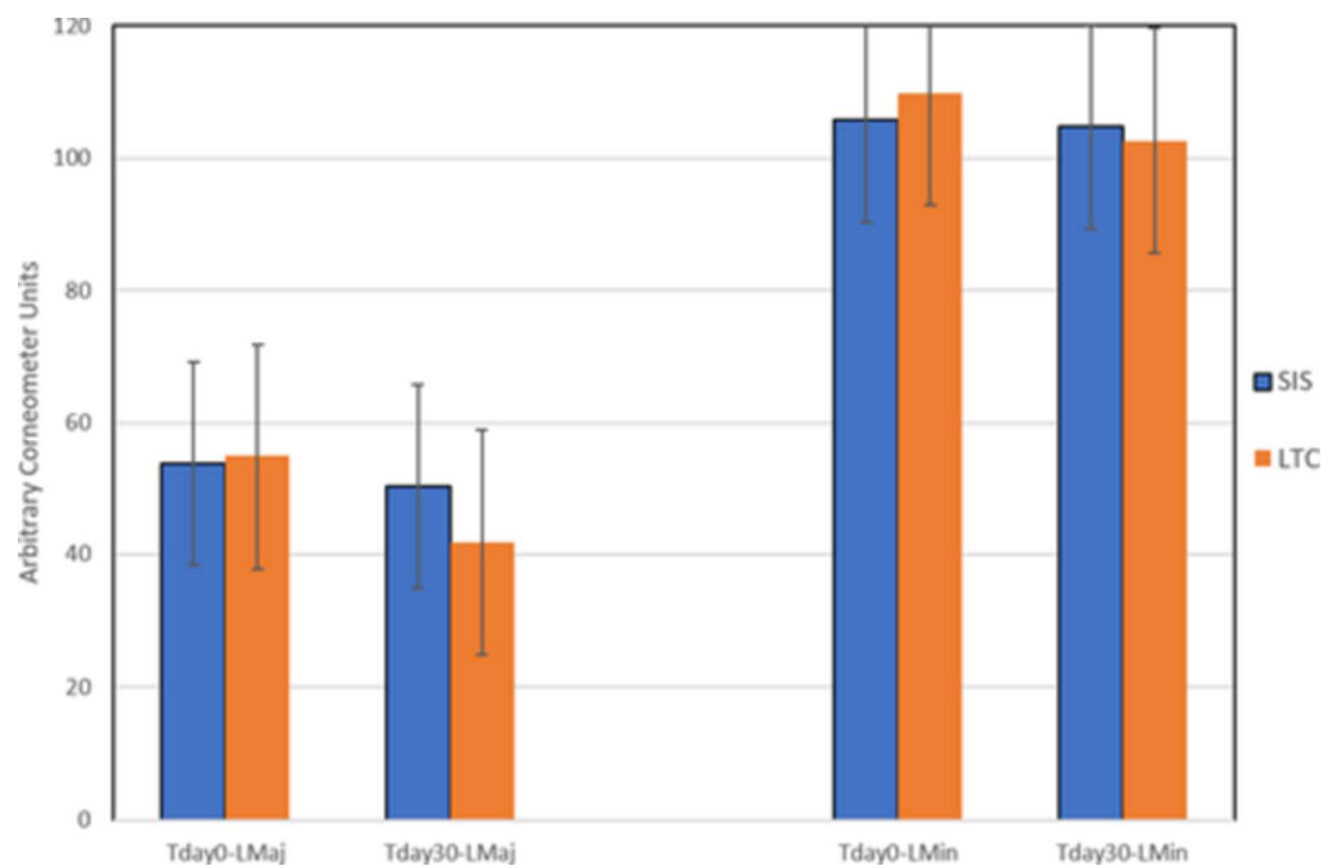

\section{Figure 1}

Vulvar skin hydration evaluated before and after treatment with SIS and LCT intimate cleanser. Values as mean between left and right side. LMaj, Labia majora; LMin, Labia minora; LTC, Lactacyd Feminine Hygiene; SIS, Saugela Hydraserum

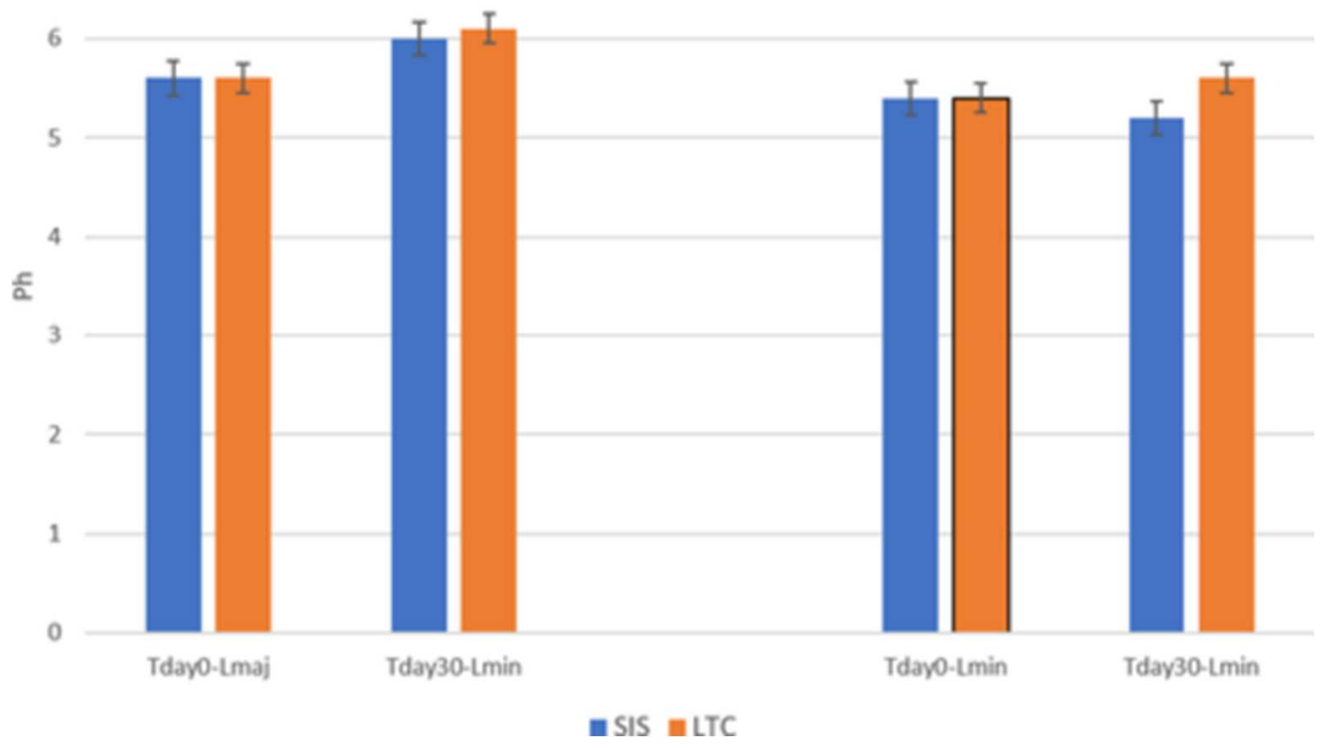

\section{Figure 2}

Vulvar $\mathrm{pH}$ comparisons before and after treatment with SIS and LCT intimate cleanser. Values as mean between left and right side. LMaj, Labia majora; LMin, Labia minora; LTC, Lactacyd Feminine Hygiene; SIS, Saugela Hydraserum 


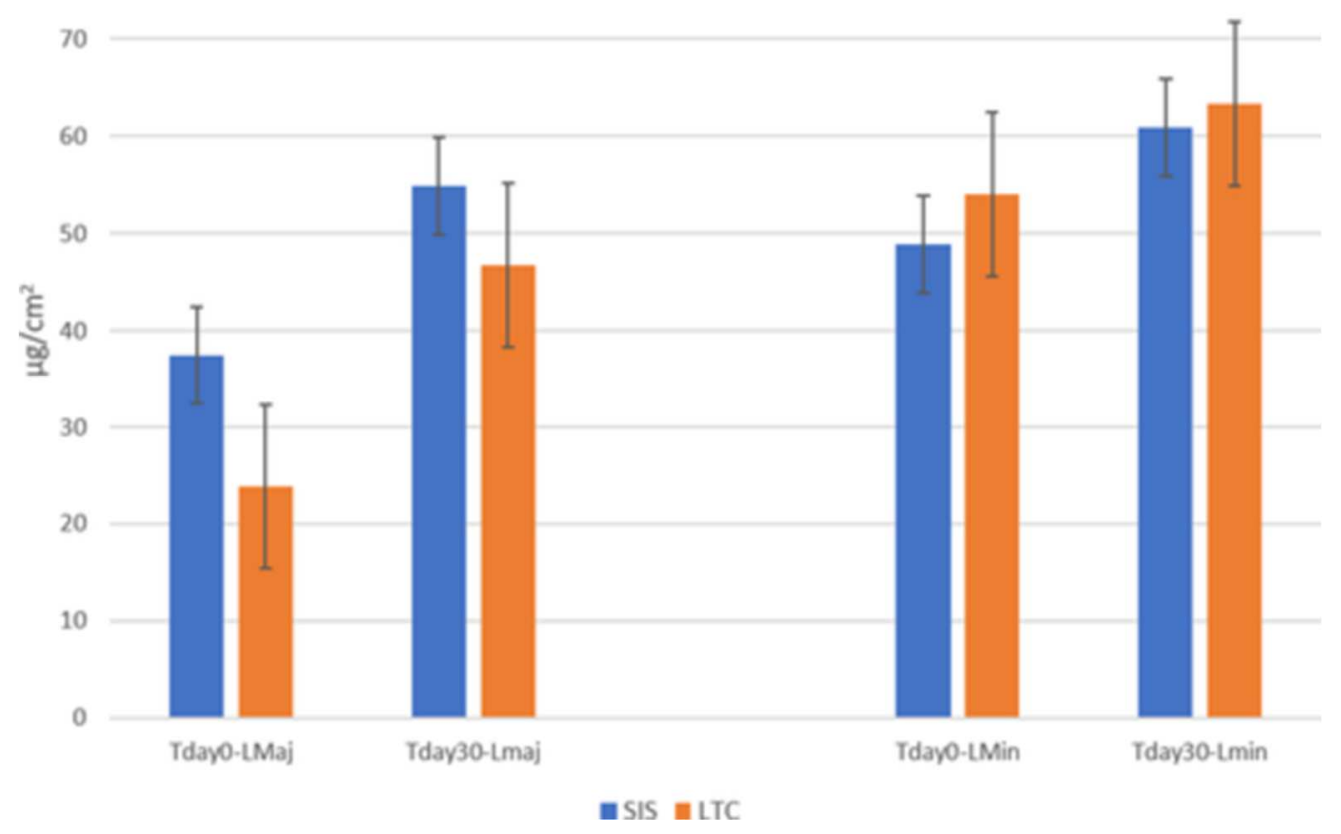

Figure 3

Vulvar skin sebum level evaluated before and after treatment with SIS and LCT intimate cleanser. Values as mean between left and right side. LMaj, Labia majora; LMin, Labia minora; LTC, Lactacyd Feminine Hygiene; SIS, Saugela Hydraserum 


\section{REFERENCES}

- 1 Van der Meijden WI, Boffa MJ, ter Harmsel WA, et al. European guideline for the management of vulval conditions. J Eur Acad Dermatol Venereol. 2017; 31(6): 925- 941.

- 2 Al-Niaimi F, Felton S, Williams J. Patch testing for vulval symptoms: our experience with 282 patients. Clin Exp Dermatol. 2014; 39: 439- 442.

- 3 Bauer A, Rödiger C, Greif C, et al. Vulvar dermatoses - irritant and allergic contact dermatitis of the vulva. Dermatology. 2005; 210: 143- 149.

- 4 Marin MG, King R, Sfameni S, Dennerstein GJ. Adverse behavioral and sexual factors in chronic vulvar disease. Am J Obstet Gynecol. 2000; 183(1): 34- 38.

- 5 Marren P, Wojnarowska F, Powel S. Allergic contact dermatitis and vulvar dermatosis. $\mathrm{Br} J$ Dermatol. 1992; 126: 52- 56.

- 6 Murina F, Benvenuti C. Randomized clinical trial on a hydrating intimate cleanser as an adjuvant in vulvar dermatosis therapy. Asian Pac J Reprod. 2013; 2(1): 42- 44.

- 7 Bahram A, Hamid B, Zohre T. Prevalence of bacterial vaginosis and impact of genital hygiene practices in nonpregnant women in Zanjan, Iran. Oman Med J. 2009; 24(4): 288- 293.

- 8 Clarys P, Clijsen R, Taeymans J, Barel AO. Hydration measurements of the stratum corneum: comparison between the capacitance method (digital version of the Corneometer CM 825 ${ }^{\circledR}$ ) and the impedance method (Skicon-200EX ${ }^{\circledR}$ ). Skin Res Technol. 2012; 18(3): 316- 323.

- 9 Youn SH, Choi CW, Choi JW, Youn SW. The skin surface pH and its different influence on the development of acne lesion according to gender and age. Skin Res Technol. 2013; 19: 131- 136.

- 10 Crowther JM. Method for quantification of oils and sebum levels on skin using the Sebumeter. Int J Cosmet Sci. 2016; 38: 210- 216.

- 11 Berardesca E, EEMCO Group. EEMCO guidance for the assessment of stratum corneum hydration: electrical methods. Skin Res Technol. 1997; 3: 126- 132.

- 12 Fevola MJ, Gentner L, Ahmad N, Librizzi JJ. Getting intimate with polymers: personal lubricants. Cosmet Toilet. 2008; 123: 59- 68.

- 13 Leo VD, Benvenuti C. Pharmacological, microbiological and clinical activity of feminine intimate cleansers based on plant extracts active principles (Saugella line). J Women's Health Care. 2015; 4: 244.

- 14 Ananthapadmanabhan KP, Moore DJ, Subramanyan K, Misra M, Meyer F. Cleansing without compromise: the impact of cleansers on the skin barrier and the technology of mild cleansing. Dermatol Ther. 2004; 17(suppl 1): 16- 25.

- 15 Fluhr JW, Kao J, Jain M, Ahn SK, Feingold KR, Elias PM. Generation of free fatty acids from phospholipids regulates stratum corneum acidification and integrity. $J$ Invest Dermatol. 2001; 117: 52- 58.

- 16 Bahamondes MV, Portugal PM, Brolazo EM, et al. Use of a lactic acid plus lactoserum intimate liquid soap for external hygiene in the prevention of bacterial vaginosis recurrence after metronidazole oral treatment. Rev Assoc Med Bras. 2011; 57(4): 415- 420. 
- 17 Royal College of General Practitioners. The management of vulval skin disorders, http://www.snhcic.org.uk/assets/cms_page_media/211/RCOG\%20\%20Vulval\%20Skin\%20.Disorders.pdf (2011, accessed December 2017).

- 18 Ananthapadmanabhan KP, Subramanyan K, Rattinger GB. Moisturising cleansers. In: JJ Leyden, AV Rawlings, eds. Skin Moisturization. Cosmetic Science and Technology Series (vol 25). New York, NY: Marcel Dekker Inc; 2002: 405- 432. 